\title{
Clinical results and complications associated with oblique lumbar interbody fusion technique
}

\author{
Cheng Cheng ${ }^{1,2 *}$, Kai Wang ${ }^{1 *}$, Can Zhang ${ }^{1}$, Hao Wu ${ }^{1}$, Fengzeng Jian ${ }^{1}$ \\ ${ }^{1}$ Department of Neurosurgery, Xuanwu Hospital of Capital Medical University, Beijing, China; ${ }^{2}$ Department of Neurosurgery, the Third Medical \\ Centre, Chinese PLA (People's Liberation Army) General Hospital, Beijing, China \\ Contributions: (I) Conception and design: H Wu, F Jian; (II) Administrative support: H Wu, F Jian; (III) Provision of study materials or patients: C \\ Cheng, K Wang; (IV) Collection and assembly of data: C Cheng, K Wang, C Zhang; (V) Data analysis and interpretation: C Cheng, K Wang; (VI) \\ Manuscript writing: All authors; (VII) Final approval of manuscript: All authors. \\ "These authors contributed equally to this work. \\ Correspondence to: Fengzeng Jian; Hao Wu. 45 Changchun Street, Beijing 100053, China. Email: jianfengzeng@xwh.ccmu.edu.cn; 13901397527@139.com.
}

Background: Oblique lumbar interbody fusion (OLIF) is a minimally invasive technique performed through the antero-oblique trajectory to address a wide range of lumbar pathologies. However, it can lead to complications. We reviewed the results of OLIF and discussed the effective methods to avoid such complications.

Methods: Seventy-nine consecutive patients who underwent OLIF between May 2016 and July 2019 were retrospectively analyzed. They were divided into three groups: stand-alone, posterior, and lateral fixation, according to whether they were followed up with auxiliary internal fixation as well as the fixation methods. Preoperative and last follow-up visual analog scale (VAS) and Oswestry Disability Index (ODI) scores were used to assess the improvement in the lower back and leg pain as well as neurological conditions. We analyzed intervertebral disc height (DH), segmental lumbar lordotic angle (SLL), lumbar lordotic angle (LL), pelvic tilt (PT), pelvic incidence-lumbar lordosis (PI-LL) mismatch, and the cross-section area (CSA) on axial magnetic resonance imaging (MRI) image in different groups. Complications, including thigh symptoms, cage subsidence, neurological injury, and vascular injury, were also noted.

Results: Seventy-nine patients were followed up postoperatively for 23.2 \pm 11.5 (range, 12-48) months. Forty-eight (61\%) patients underwent stand-alone surgery (without fixation), 15 (19\%) patients underwent supplemental percutaneous pedicle screw fixation (posterior fixation), and 16 (20\%) patients underwent lateral vertebral instrumentation (lateral fixation). In all three groups, the VAS score and the ODI score had significantly decreased at the final follow-up compared to pre-operation. The DH, SLL, LL, CSA, PT, and PI-LL mismatch had also improved by final follow-up. The most common approach-related complication was thigh symptoms. Of the 79 patients, ipsilateral transient psoas paresis occurred in $9(11.4 \%)$, ipsilateral transient quadriceps weakness in $2(2.5 \%)$, and groin/thigh numbness and pain in 17 (21.5\%). Cage subsidence occurred in $8(10.1 \%)$ patients, including five cases of grade 0 , one of grade I, and two of grade II. Three $(3.8 \%)$ patients in this study had a vascular injury.

Conclusions: OLIF is a minimally invasive and effective technique for dealing with degenerative lumbar diseases. However, it should also be noted that this approach carries risks of complications.

Keywords: Oblique lumbar interbody fusion (OLIF); lateral retroperitoneal approach; complications; degenerative lumbar disease

Submitted Mar 03, 2020. Accepted for publication Oct 10, 2020.

doi: 10.21037/atm-20-2159

View this article at: http://dx.doi.org/10.21037/atm-20-2159 


\section{Introduction}

Lumbar interbody arthrodesis is a safe, effective surgical procedure to treat lumbar disc herniation, degenerative disc disease, instability in lumbar spondylolisthesis, spinal deformity, and discogenic back pain. It can increase fusion rates and correct coronal and sagittal alignment (1).

Minimally invasive approaches have been increasingly applied over the years, each with their advantages and disadvantages. Posterior lumbar interbody fusion (PLIF) and transforaminal lumbar interbody fusion (TLIF) have demonstrated reasonable fusion rates and clinical results. Nonetheless, compared to anterior lumbar interbody fusion (ALIF), PLIF and TLIF only allow smaller cage sizes, incomplete anterior release, and also increase the risk of nerve root traction injury (2). ALIF provides efficient access to the anterior spinal column, allows comprehensive intervertebral disc clearance, and permits a large cage to restore lordosis and disc height $(\mathrm{DH})$. It also increases the neuroforaminal volume and indirectly decompresses existing nerve roots (3). However, ALIF is also associated with comparatively high rates of approach-related complications, such as injury to the ureters, major retroperitoneal vessels, peritoneum, ilioinguinal and iliohypogastric nerves, and autonomic nervous system. It also involves a risk of causing retrograde ejaculation associated with superior hypogastric plexus injury (4). Extreme lateral interbody fusion (XLIF) was first introduced by Pimenta and later popularized by Ozgur et al. in 2006 (5). XLIF avoids the paraspinal muscle and longitudinal ligament dissection required by TLIF and PLIF and does less direct harm to the abdominal viscera than ALIF (6). XLIF allows less vascular injury, better access, shorter operation time, larger cage placement, less bleeding, and faster recovery (7). Nevertheless, lateral access is obstructed at L5/S1 owing to the overlap of the iliac crest. XLIF is also associated with postoperative thigh symptoms of pain, numbness, or weakness, even with a meticulous surgical technique and neuromonitoring, as it passes through the psoas. Moreover, real-time electromyography monitoring is essential when performing XLIF surgery owing to the lumbar plexus course through the psoas (8).

The oblique lumbar interbody fusion (OLIF) approach to the lumbar spine via the corridor between the psoas major and the aorta has been developed to reduce the procedurerelated complications (5). It avoids the peritoneum and causes fewer dural injuries compared to anterior and posterior interbody fusion techniques. It is a wellaccepted alternative to anterior, posterior, and transpsoas procedures for lumbar interbody fusion. Additionally, neuromonitoring is unnecessary because the lumbar plexus remains intact. OLIF has theoretical advantages such as indirect decompression of neural structures, preservation of ligaments, a large footprint to span the apophyseal ring, and a satisfactory fusion rate. However, no surgical technique is a panacea for ensuring perfect safety, and this study aims to explore the clinical effect and associated complications with OLIF. We have presented the following study in accordance with the STROBE reporting checklist (available at http:// dx.doi.org/10.21037/atm-20-2159).

\section{Methods}

The study was conducted per the Declaration of Helsinki (as revised in 2013). The study was approved by the institutional research ethics committee of the Xuanwu Hospital of Capital Medical University \{No.[2018]027\}, which waived the requirement for informed patient consent because of the retrospective nature of the analysis.

We retrospectively analyzed 79 patients who underwent OLIF surgery (stand-alone or with posterior percutaneous screws/lateral instrumentation), from May 2016 to July 2019. The stand-alone procedure used no supplemental posterior instrumentation. Demographic information was collected for all patients (Table 1). The operation time was measured as the time from initial incision to incision closure. Patients' general and neurological conditions were evaluated at admission and final follow-up using the visual analog scale (VAS) and the Oswestry Disability Index (ODI). All patients underwent four-view X-ray, computed tomography (CT), magnetic resonance imaging (MRI), or computed tomographic angiography (CTA) examinations preoperatively and postoperatively.

The inclusion criteria were the following: (I) patients with chronic back pain and/or leg pain, with worsening neurologic deficits and neurogenic intermittent claudication; (II) patients with diseases including lumbar degenerative disc herniation, mild spondylolisthesis (grade I or II), spinal stenosis, lumbar degenerative scoliosis/kyphosis, adjacent segment disease, and spinal spondylolysis with or without vertebral slippage; and (III) failure of previous conservative treatment for a minimum of 6 months. We excluded patients with an active infection, spinal tumor, trauma, severe osteoporosis, peritoneal operation history, high-grade spondylolisthesis, severe lumbar spinal canal stenosis requiring direct decompression, and spontaneous facet joint fusion of the addressed segment. Stand-alone OLIF or combined 
Table 1 Demographic characteristics

\begin{tabular}{|c|c|}
\hline Characteristics & Value \\
\hline Total & 79 \\
\hline Sex (male/female) & $34 / 45$ \\
\hline Age (years), mean \pm SD [range] & $67 \pm 10[37-86]$ \\
\hline Follow-up (months), mean \pm SD [range] & $23.2 \pm 11.5[12-48]$ \\
\hline $\mathrm{BMI}\left(\mathrm{kg} / \mathrm{m}^{2}\right)$, mean $\pm \mathrm{SD}$ [range] & $25.9 \pm 3.6[16.6-35.9]$ \\
\hline $\mathrm{EBL}(\mathrm{mL})$, mean $\pm \mathrm{SD}$ [range] & $142.5 \pm 45.8[142-300]$ \\
\hline Operation time (min), mean \pm SD [range] & $197.7 \pm 129(71 \pm 637)$ \\
\hline $\begin{array}{l}\text { Length of hospitalization (days), mean } \pm \\
\text { SD [range] }\end{array}$ & $7.8 \pm 3.5[3-28]$ \\
\hline \multicolumn{2}{|l|}{ Diagnosis } \\
\hline Degenerative disc herniation & 36 \\
\hline Degenerative spondylolisthesis & 12 \\
\hline Isthmic spondylolisthesis & 3 \\
\hline Lumbar stenosis & 9 \\
\hline Lumbar degenerative scoliosis/kyphosis & 11 \\
\hline Adjacent segment disease & 8 \\
\hline \multicolumn{2}{|l|}{ Concomitant diseases } \\
\hline Hypertension & 43 \\
\hline Diabetes mellitus & 15 \\
\hline Coronary artery disease & 9 \\
\hline \multicolumn{2}{|l|}{ Number of fusion levels } \\
\hline T12/L1 & 2 \\
\hline L1/L2 & 5 \\
\hline L2/L3 & 31 \\
\hline L3/L4 & 49 \\
\hline L4/L5 & 57 \\
\hline Stand-alone surgeries & 48 \\
\hline \multicolumn{2}{|l|}{ Instrumented surgeries } \\
\hline Percutaneous pedicle screws & 15 \\
\hline Lateral vertebral instrumentation & 16 \\
\hline
\end{tabular}

BMI, body mass index; EBL, estimated blood loss; SD, standard deviation.

OLIF (posterior percutaneous pedicle screw fixation or lateral instrumentation) was performed. A supplementary posterior or lateral instrumentation was performed if any of the following conditions were true: (I) dual-energy X-ray absorptiometry (DEXA) indicated $t$ value $<1.0$, (II) a body mass index $>30 \mathrm{~kg} / \mathrm{m}^{2}$, (III) negligent intraoperative endplate damage, (IV) grade II spondylolysis (9).

The following complications were considered to be approach-related: spinal dura mater tear, abdominal vascular injury, ureteral injury, ipsilateral psoas paresis, ipsilateral quadriceps weakness, sympathetic chain symptom, screw malposition, segmental vascular injury, cage sedimentation or shifting, wound infection, peritoneal laceration, left lower abdominal pain, incomplete ileus, contralateral nerve root injury, vertebral fracture, reoperation, right psoas major hematoma, pseudarthrosis, and pseudohernia. Non-approachrelated complications included urinary system complications, respiratory complications (pneumonia), and delirium.

Fusion criteria included bony trabeculation across the intervertebral level and no bony lucency at the vertebral body junction (10). The subsidence of an interbody fusion cage was also detected. Subsidence grading is defined as the percentage of vertebral body or disc space collapse compared with the immediate postoperative images (11). Marchi et al. (12) analyzed radiographic graft subsidence following stand-alone LLIF. They proposed a grading scale to describe the degree of subsidence, with low-grade being grades 0 and I ( $0-24 \%$ and $25-49 \%$, respectively) and high-grade being grades II and III (50-74\% and $75-100 \%$, respectively). The CT scans and standing lateral lumbar spine radiographs were evaluated by an independent radiologist and the operation team according to these criteria.

\section{Surgical technique}

The patient was placed in a correct right-sided lateral decubitus position. The fluoroscopic examination was used to confirm the disc level, which was marked on the skin. A 3-4 cm skin incision was made for a single-level fusion, centered on the target segment and parallel to the external oblique muscle, followed by blunt dissection of the internal oblique muscle fascia and transverse abdominis along the direction of the muscle fibers. For multilevel fusions, a sliding window technique was applied to access the disc spaces without expansion of the initial incision. After blunt dissection of the retroperitoneal space, the peritoneum and vascular structures were mobilized anteriorly with posterior retraction of the psoas major. Then, the intervertebral disc was exposed through a corridor between the aorta and the psoas, and the retractor system was docked. Neuromonitoring was not conducted because no dissection 
of the muscular fibers was done. Discectomy was performed sequentially; the cartilaginous endplate was removed to expose the subchondral bone. A proper sized cage (DePuy Synthes, Raynham, MA, USA) was packed with allogeneic bone graft and hydroxyapatite. The implantation started from the lateral oblique direction, proceeding through the true lateral direction using the rotation maneuver. Fluoroscopy was applied to confirm that the implants and instruments were in a proper position. To avoid cage subsidence and loss of intervertebral space, lateral or posterior supplemental instrumentation was subsequently applied. Lateral screws were placed into the vertebral bodies without repositioning or draping, and the entrance points were kept proximal to the adjacent endplate to avoid segmental vessel injury. For patients who underwent posterior percutaneous pedicle screw fixation, they were repositioned to prone decubitus and then performed based on the routine procedure.

\section{Statistical analysis}

Statistical analyses were performed using the Statistical Package for the Social Sciences (SPSS Version 24, IBM Corp., Armonk, NY, USA). The patients were divided into three groups: stand-alone, posterior, and lateral fixation. The index of the preoperative and final follow-up clinical results was compared. For the multilevel cases, the average score was calculated: VAS, ODI, intervertebral DH (the average of the anterior and posterior $\mathrm{DH}$ : millimeter $\mathrm{mm}$ ), segmental lumbar lordotic angle (SLL) and lumbar lordotic angle (LL), pelvic tilt (PT), pelvic incidence-lumbar lordosis (PI-LL) mismatch, and the cross-section area (CSA) on axial MRI image. The continuous data were reported as a mean \pm standard deviation. The categorical data were described as frequencies and percentages. A Chi-square test was used to analyze categorical variables. The comparisons between preoperative and postoperative parameters within groups were performed by the paired Student's $t$-test, and the Kruskal-Wallis test was used to compare the three groups. Statistical significance was set at an alpha level of 0.05 .

\section{Results}

Seventy-nine patients ( 34 men, 45 women) were included in this cohort, and 144 levels were treated. They were followed postoperatively for $23.2 \pm 11.5$ (range, 12-48) months. Patients' demographic characteristics are shown in Table 1. Forty-eight (61\%) patients underwent stand-alone surgery,
$15(19 \%)$ patients underwent supplemental percutaneous pedicle screw fixation (posterior fixation), and 16 (20\%) patients underwent lateral vertebral instrumentation (lateral fixation). Satisfying results were obtained in selected patients (Figures 1,2). An average of 1.8 levels were treated per patient (range, T12-L5). The most frequently treated levels were L4/L5 (29.7\%), followed by L3-L4 (25.2\%) and L2-L3 (16.1\%). In the three groups, the mean VAS scores for back pain and leg pain, as well as the ODI score, decreased significantly by the final follow-up visit compared with pre-operation measurements. Meanwhile, there was no statistical difference in the index among the three groups (seen Table 2). The DH, SLL, LL, CSA, PT, and PI-LL mismatch improved after the operation. The improvements were statistically significant when compared to preoperation; however, the indexes showed no statistically significant differences among the groups in this study (see Table 3).

The most common approach-related complication (Table 4) was thigh symptoms: ipsilateral transient psoas paresis occurred in 9 of $79(11.4 \%)$ patients, ipsilateral transient quadriceps weakness in 2 of $79(2.5 \%)$ patients, and groin/thigh numbness and/or pain in 17 of $79(21.5 \%)$ patients. Most complications disappeared after 3 months, while in one case of quadriceps weakness, the complication resolved 6 months postoperatively. In this study, no retrograde ejaculation, peritoneal laceration, incomplete ileus, or ureter injuries occurred. Cage subsidence occurred in eight patients, including five cases of grade 0 , and one case of grade I (three grade 0 and one grade I in the standalone group, one grade 0 in posterior fixation group, and one grade 0 in the lateral fixation group). These patients did not undergo reoperation owing to symptom improvement. There were also two cases of grade II in the stand-alone group, which occurred in three levels addressed segments. Their back pain worsened, and they had to undergo posterior percutaneous instrumentation 6 months postoperatively.

Three patients in this study suffered a vascular injury. One patient underwent intervascular embolization for vascular injury, retroperitoneal bleeding, and subsequent shock (Figure 3). She also underwent immediate endovascular interventional treatment and hematoma evacuation, following which she showed good recovery. Two segmental vascular lesions were identified during the operation and were controlled with electrocoagulation hemostasis. Another patient suffered sharp pain when turning from prone to supine, and MRI revealed a disc 

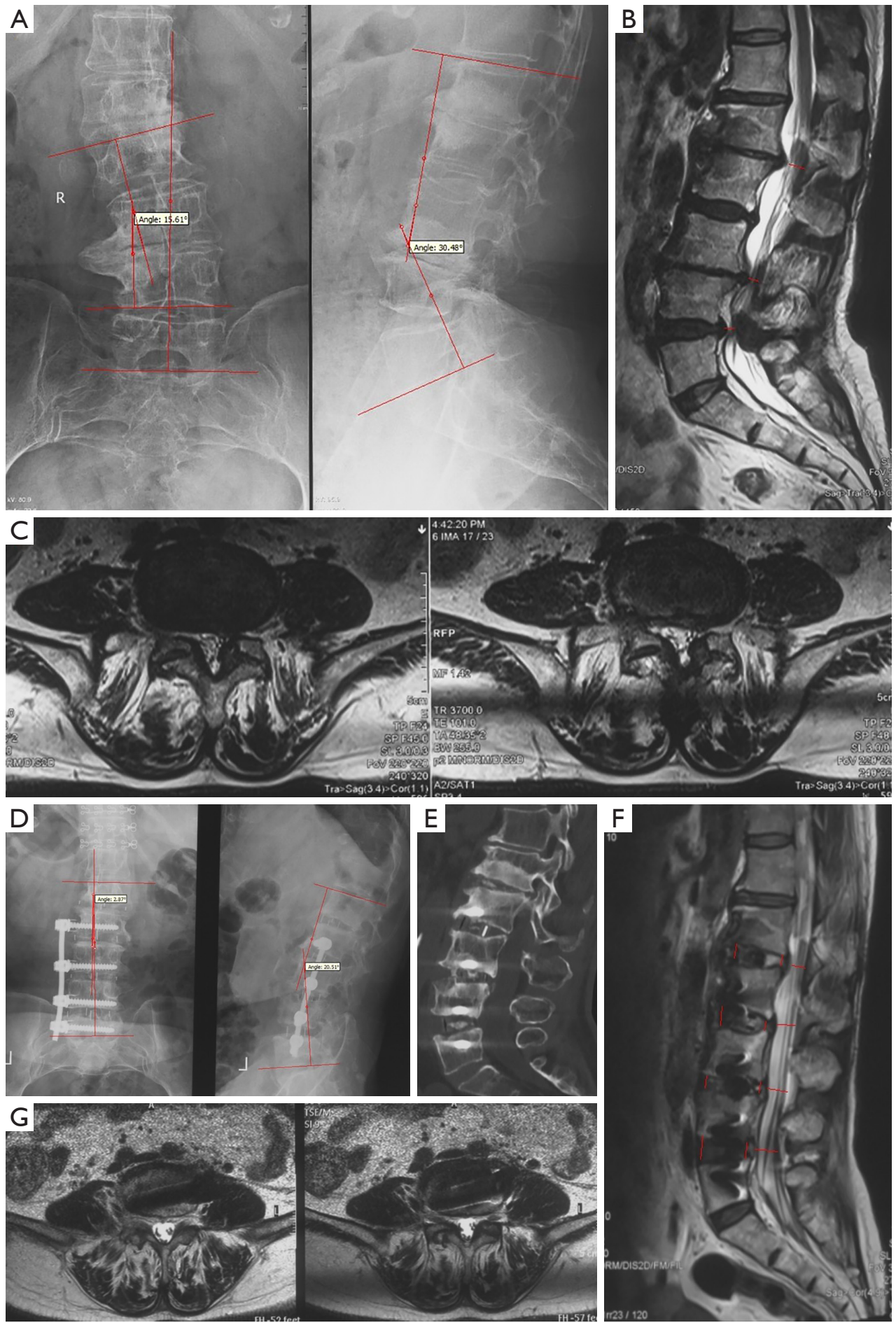

Figure $1 \mathrm{~A}$ female, 56 years old, had the chief complaint of back pain and intermittent claudication for two years. Preoperative images examination indicated she had segmental lumbar scoliosis and stenosis, manifested as coronal spinal imbalance (A: fluoroscopy results), smaller central spinal canal diameter from L1-2 to L4-5, and disc height loss (B and C: sagittal global lumbar and L3-4 axial MRI results). Postoperative images showed that coronal imbalance was modified from $15.61^{\circ}$ to $2.87^{\circ}$ after applying lateral instrumentation (D). The inserted proper sized cage from L1-2 to L4-5, four levels total, restored the disc height, amplified the central canal area, and achieved indirect decompression (E,F,G). Six-month follow-up CT revealed that the trabecular bone was seen between the cage and vertebrates. 

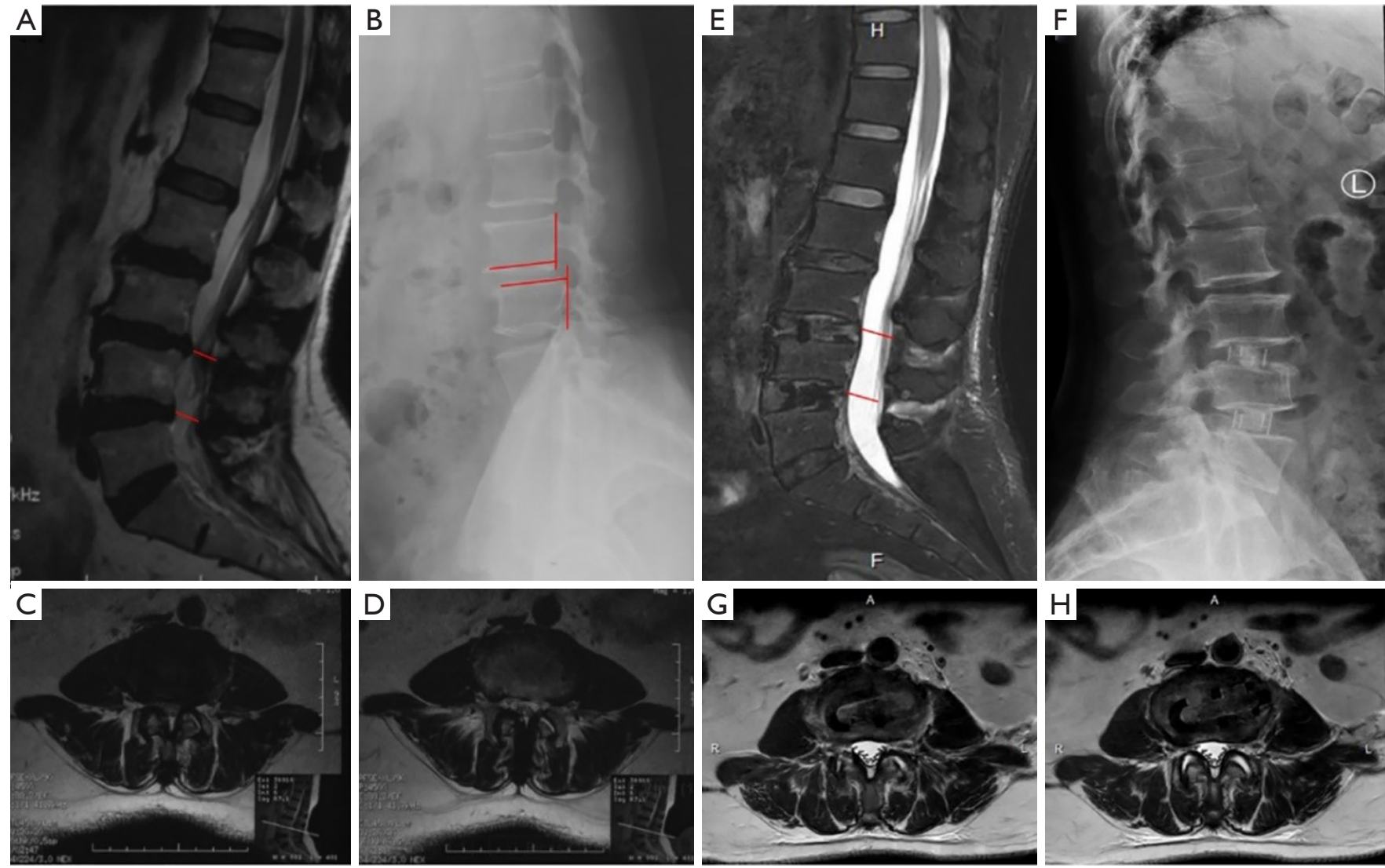

Figure 2 A female, 63 years old, suffered back pain for 1 year. Preoperative MRI indicated L3-4 and L4-5 stenosis (A,C,D). X-ray examination showed L3 spondylolisthesis (B, grade I). After the stand-alone OLIF in the two segments, L3 vertebrae spondylolisthesis got corrected, intervertebral height was rebuilt (F), and the central canal region was dilated (E,G,H). MRI, magnetic resonance imaging; OLIF, oblique lumbar interbody fusion.

Table 2 Preoperative and final follow-up low back and leg pain scores and ODI

\begin{tabular}{lccc}
\hline Variables & Stand alone & Posterior fixation & Lateral fixation \\
\hline Low back pain VAS & & & P value \\
Pre-low back pain VAS & $5.08 \pm 1.91$ & $4.53 \pm 2.20$ & $5.19 \pm 2.43$ \\
Final follow-up low back pain VAS & $2.69 \pm 1.32$ & $1.46 \pm 1.42$ & $2.01 \pm 1.41$ \\
P value & $<0.001$ & 0.001 & $<.449$ \\
Leg pain VAS & & & $2.81 \pm 1.11$ \\
Pre-leg pain VAS & $3.89 \pm 2.16$ & $4.13 \pm 2.19$ & $1.13 \pm 0.81$ \\
Final follow-up leg pain VAS & $1.56 \pm 1.12$ & $1.88 \pm 1.32$ & $<0.001$ \\
P value & $<0.001$ & 0.001 & 0.106 \\
ODI & & $32.80 \pm 10.81$ & $28.06 \pm 13.59$ \\
Pre-ODI & $30.06 \pm 15.32$ & $16.87 \pm 7.52$ & $15.44 \pm 13.96$ \\
Final follow-up ODI & $14.96 \pm 9.15$ & 0.001 & 0.009 \\
P value & $<0.001$ & & 0.441
\end{tabular}


Table 3 Changes in preoperative and postoperative DH, SLL, CSA, LL, PT, PI-LL

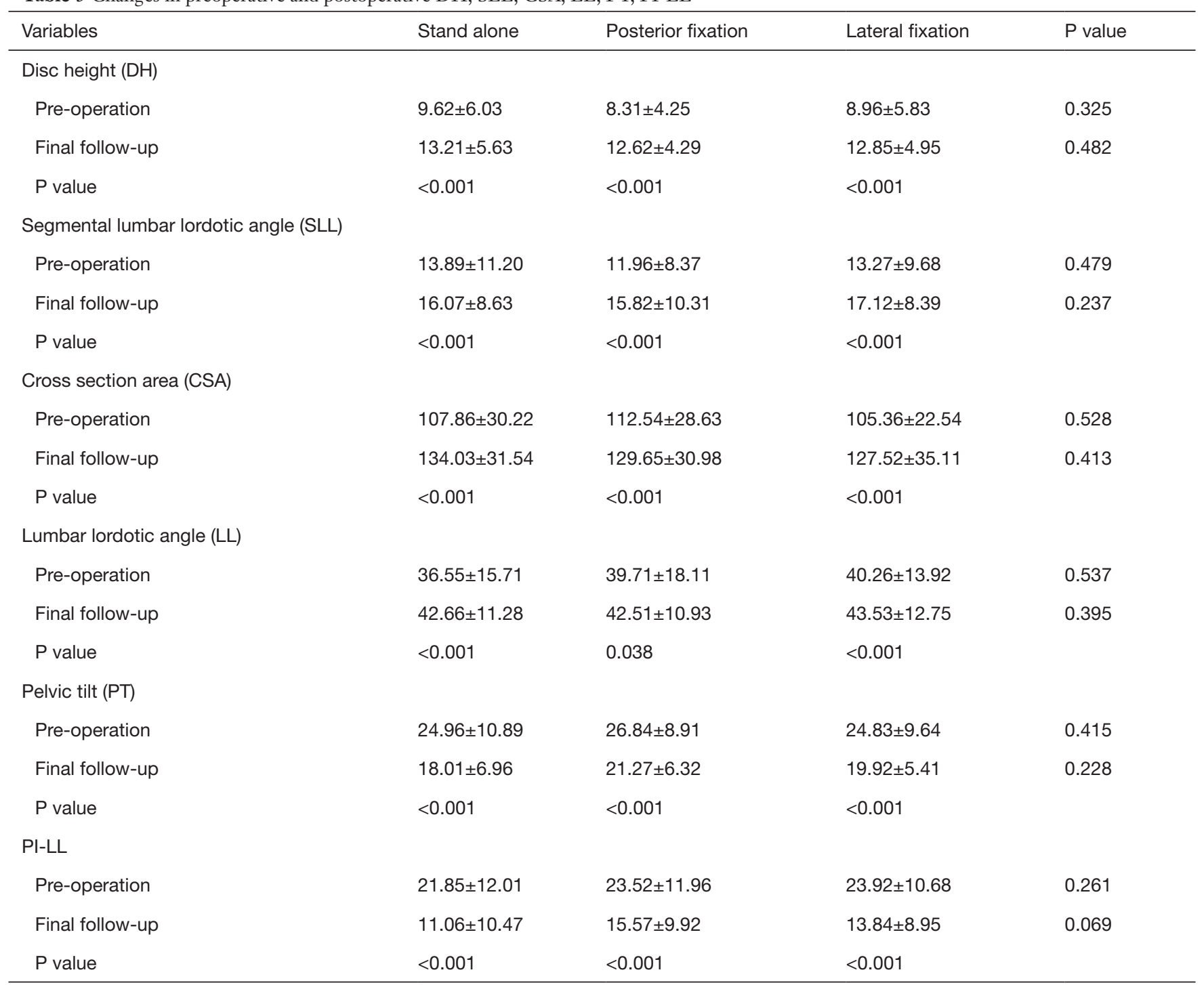

PI-LL, pelvic incidence-lumbar lordosis.

residual was compressing the contralateral nerve root (Figure 4). Psoas hematoma, vertebral endplate injury, and retroperitoneal effusion were also noted (Figures 5-7, respectively).

\section{Discussion}

OLIF helps preserve the psoas muscle and does not require dissection. This theoretically reduces postoperative thigh pain $(13,14)$. This approach has minimal peritoneal retraction, direct disc space visualization, a larger cage, less blood loss, combined indirect and direct decompression, and no need for neuromonitoring (15). It has been previously reported that the L4-L5 level could also be reached in cases with a high-riding iliac crest for a slightly anterior OLIF approach, and it was also possible to access L5-S1 in the lateral position (16). Furthermore, OLIF has broad indications, a low risk of post-treatment trauma, excellent stability, and short hospitalization time. The advantage of stand-alone OLIF in reducing operation time and injury has further promoted its clinical application (17). However, no technique is a panacea, and the complications cannot be fully avoided.

In this study, the most frequent complications were 
Table 4 Complications and number of patients

\begin{tabular}{|c|c|}
\hline Complications & No. of patients $(n=79)$ \\
\hline \multicolumn{2}{|l|}{ Approach-related complications } \\
\hline Abdominal vascular injury & 1 \\
\hline Spinal dural tear & 0 \\
\hline Ureteral injury & 0 \\
\hline \multicolumn{2}{|l|}{ Thigh symptoms } \\
\hline Psoas paresis & 9 \\
\hline Quadriceps weakness & 2 \\
\hline Groin/anterior thigh numbness/pain & 17 \\
\hline Sympathetic chain symptom & 1 \\
\hline Screw malposition & 0 \\
\hline Segmental vascular injury & 2 \\
\hline Cage subsidence & 8 \\
\hline Wound infection & 2 \\
\hline Peritoneal laceration & 0 \\
\hline Left lower abdominal pain & 3 \\
\hline Incomplete ileus & 0 \\
\hline Contralateral nerve root injury & 1 \\
\hline Vertebral fracture & 0 \\
\hline Right psoas major hematoma & 3 \\
\hline Pseudarthrosis & 0 \\
\hline Pseudohernia & 1 \\
\hline \multicolumn{2}{|l|}{ Non-approach-related complications } \\
\hline Urinary system complications & 0 \\
\hline Respiratory complication (pneumonia) & 2 \\
\hline Delirium & 1 \\
\hline
\end{tabular}

groin/thigh numbness and/or pain, and ipsilateral iliopsoas weakness. The high-rate incidences of groin/ thigh complications in this cohort occurred mainly in the initial period of learning and applying this new technique. Thigh symptoms, including thigh weakness and knee extensor muscle weakness, abnormal sensation of dysesthesia, paresthesia, burning, and pain reportedly result from direct nerve injury and/or indirect compressive neuropathy (18). Transient thigh symptoms are always experienced postoperatively and could be as high as $62.7 \%$ in XLIF cohorts (19). For OLIF, prior studies reported rates ranging from $1.3 \%$ to $21.4 \%(1,13,17)$, but these are likely underestimated because only motor dysfunction was described and not sensory abnormalities. Adverse postoperative neurological effects are still a significant concern despite intraoperative nerve monitoring (20).

While OLIF may decrease neurological injury by preserving psoas and avoiding the lumbar plexus, direct and/or indirect harm to the lumbar plexus nerves producing transient or permanent neurological damage can still occur (21). For example, during the separation of internal oblique muscle and transverse muscle, the iliohypogastric nerve or the ilioinguinal nerve may be injured. The genitofemoral nerve or the sympathetic chain could also be damaged when pulling or stripping the psoas (22). If there is an incorrect invasion of the contralateral canal or the intervertebral foramen after cage insertion, the caudal nerve or the nerve root can get injured (13). Moreover, a high degree of prolonged nerve compression or excessive stretching of the psoas can lead to ischemia of the involved nerve and functional failure (20). The psoas major hematoma compresses the ipsilateral or contralateral lumbar plexus. This combination of neuropathy, mechanical compression, and stripping, therefore, can lead to thigh symptoms. Long-term and high-power compression and retraction of the psoas major muscle can also induce the bad paresthesia.

According to different lumbar levels, surgeons must be familiar with the local anatomy and carry out the operation in neurologically safe zones $(9,23,24)$. The iliohypogastric and ilioinguinal nerves should be protected while the abdominal muscle layers are being separated. Clinicians should also be wary of psoas hematoma and must try to avoid it during contralateral annulus release. Protection methods also include intermittent retractor release and cage insertion orthogonal to the vertebral body in the sagittal view to prevent it from impinging the contralateral nerve root or being advanced into the vertebral canal $(25,26)$.

The goal of interbody fusion is complete incorporation of the fusion and restoration of DH. Subsidence may result in loss of disc space height and angle correction, jeopardizing the indirect decompression of nervous structures and the anatomic quality of the fusion. Furthermore, loss of lordosis due to anterior subsidence carries the risk of adjacent segment degenerative disease (27). Factors such as osteoporosis, improper operative practices, endplate violation, cage sizes, vertebroplasty, posterior pedicle screw fixation, and mono or multiple segmental fusion could affect subsidence and impact the clinical results (28).

Stand-alone OLIF without posterior instrumentation has 

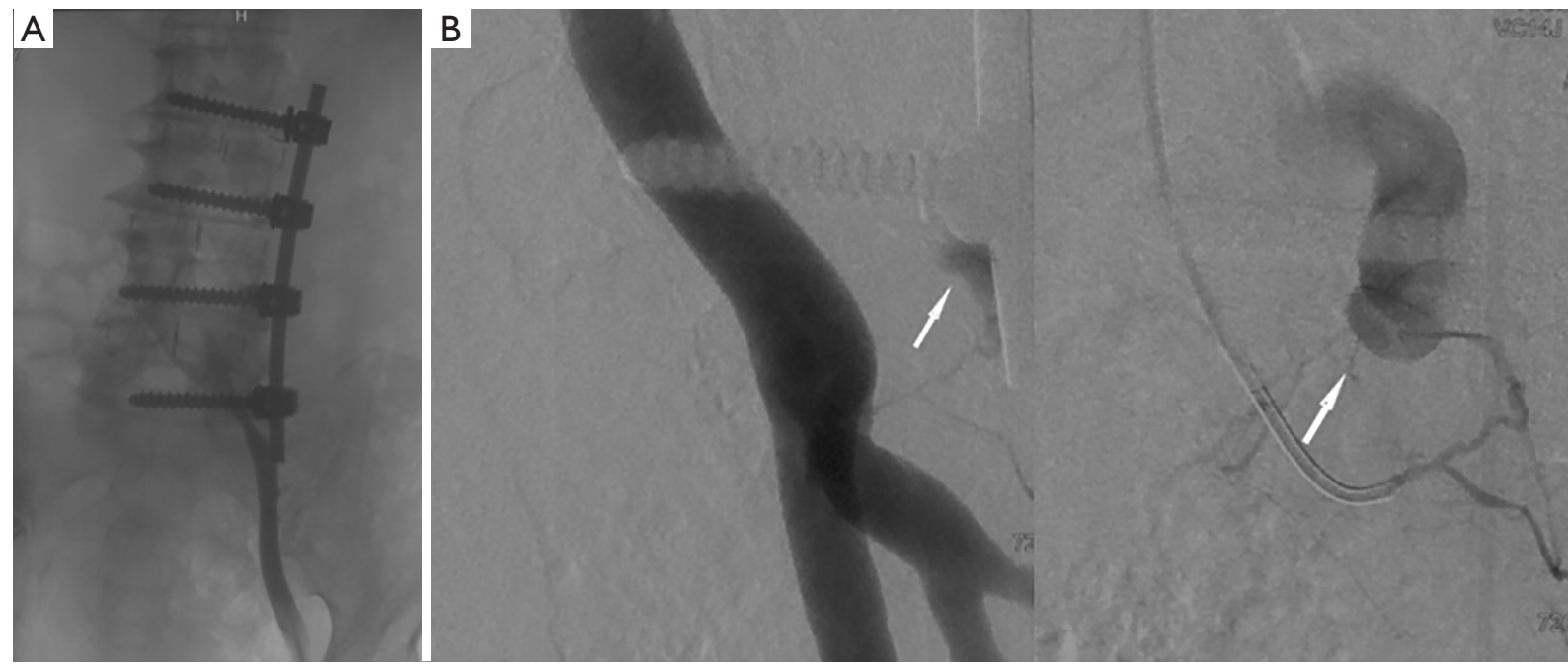

Figure 3 Intravascular embolization and hematoma evacuation were done for a retroperitoneal hematoma and shock case. Digital subtraction angiography revealed the internal iliac artery and lateral fixation (A); bleeding point at a branch of the internal iliac artery (white arrow middle); embolization with Onyx (white arrow right) (B).
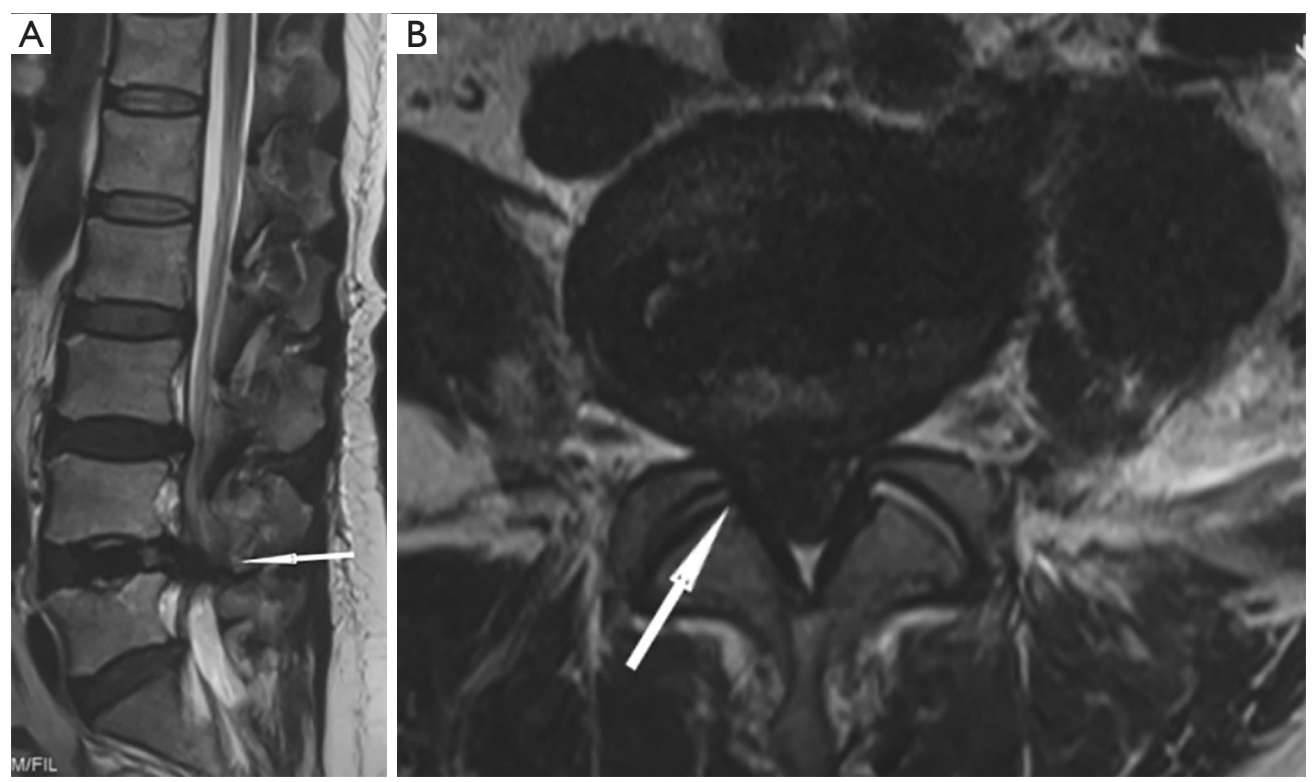

Figure 4 Sharp pain occurred when the patient was moved from the operation vehicle to the ward bed about 1 hour after surgery. A residual intervertebral disc intruded the contralateral foraminal and compressed the nerve root (lumbar T2-weighted MRI white arrow; A,B), then a revision surgery was applied from the posterior trajectory. MRI, magnetic resonance imaging.

been used in selected patients (29). Our study indicated that in $75 \%$ of cases, the incidence of cage subsidence was grade 0 , and was highest in the stand-alone group. Two cases of grade II required posterior percutaneous instrumentation. The DEXA examination was recommended for patients over age 50 with suspected osteopenia or osteoporosis. OLIF with supplemental posterior or lateral fixation at the initial surgery is preferable in patients with osteoporosis. Supplemental posterior pedicle screws can provide threecolumn fixation, maximal load sharing, and support. A 


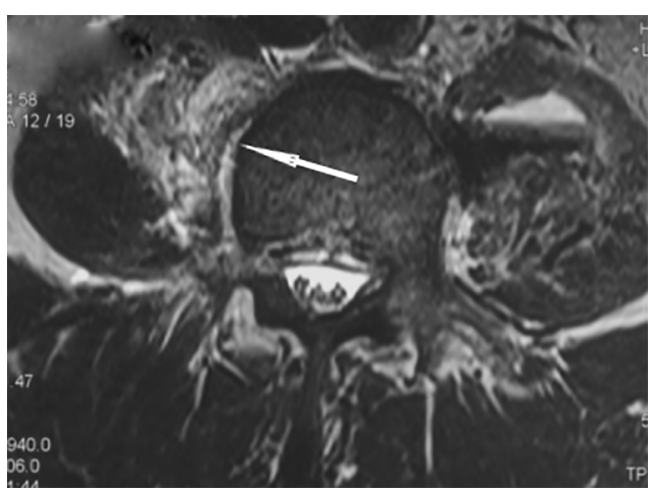

Figure 5 Right psoas hematoma (white arrow) was found and accompanied back and groin pain; however, the symptom remarkably relieved 3 months later with conservative treatment.

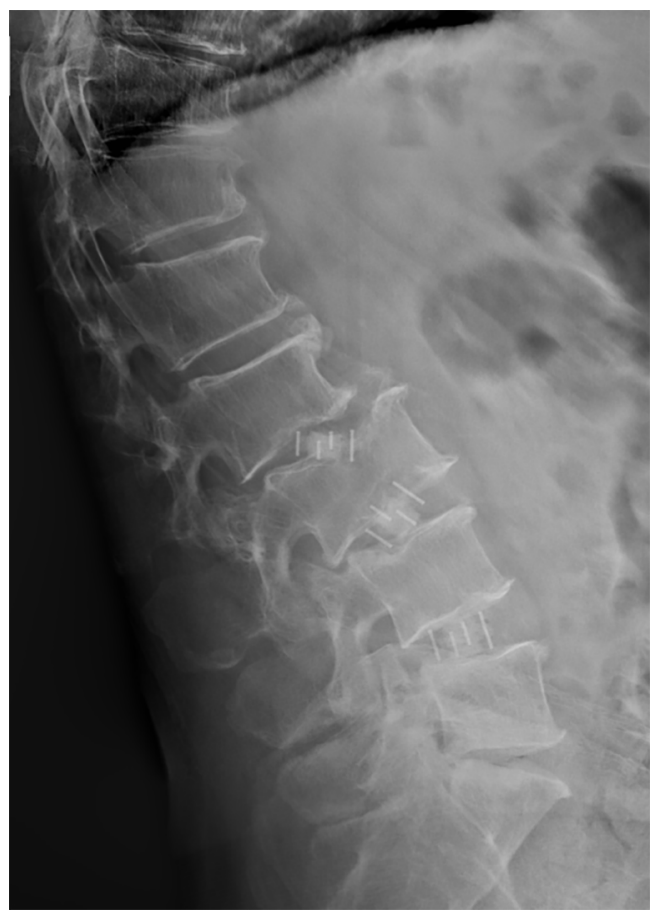

Figure 6 The intraoperative vertebral endplate was injured due to the placement of the intervertebral cage in the wrong direction.

proper sized cage could also be used to increase contact with the cortical bone and apophyseal ring to prevent subsidence (30). Meticulous endplate preparation is highly essential, and gentle blunt force is needed to open the intervertebral disc; otherwise, endplate violation would weaken the axial loading stress on the vertebral bone for the graft directly in contact with the cancellous bone (11). As

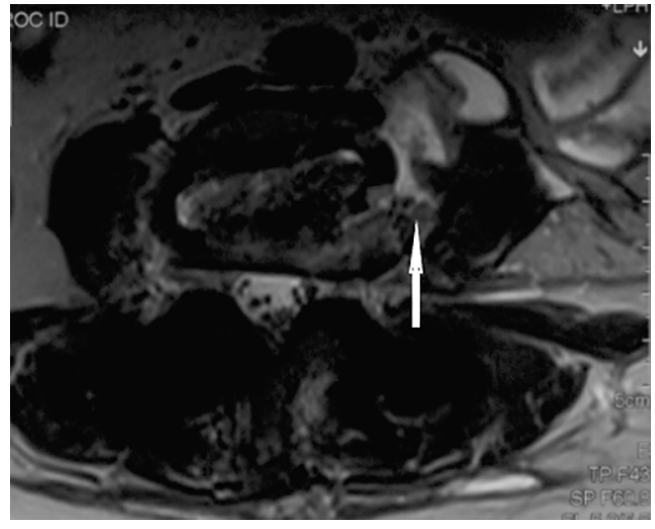

Figure 7 Retroperitoneal effusion (white arrow) accumulated at the operative corridor and compressed the psoas major, resulted in $3 / 5$ muscle strength of the ipsilateral thigh. Reopened the wound, aspirated the hydroma, and quickly the muscle strength improved to $4 / 5$.

illustrated in Figure 6, the bony endplate can be injured due to excessive reaming of the endplate cartilage.

During the operation, the cage should be inserted into the intervertebral space parallel to the endplate to avoid endplate violations (28). The lumbar lordosis, scoliosis, and vertebral rotation can change the original direction of the intervertebral space. Thus, the fluoroscopy should always be adjusted to find the exact direction of intervertebral space and be used to guide cage insertion (31). Furthermore, it is unclear whether the low-grade cage subsidence is true subsidence without accompanying symptoms. This needs to be investigated further when following up or during the fusion process of the cage and the vertebral bone $(10,32)$.

Operative vascular injury can occur on the ipsilateral or contralateral side. However, its incidence in our study was acceptable (3 cases, 3.8\%) compared to previous reports. Silvestre et al. (13) reported iliac vessel injury in $1.1 \%$ of 179 patients who underwent OLIF. Assina et al. (33) described a case fatality due to great vessel injury, caused by the detachable blade following an XLIF approach. Zeng et al. (28) also reported a $2.98 \%$ incidence of vascular injury in 7 OLIF procedures. Preoperative evaluation of the major vessels and their anatomical variations is vital to prevent catastrophic complications. MRI and CTA examination are recommended to analyze the relationship between the vessels, the psoas, and the bifurcation of the aorta iliac vessels. The surgeons should be familiar with the patients' anatomy and variations and take care not to violate the anterior longitudinal ligament to avoid possible vascular injury. They should also check for bleeding when 
removing the self-retractor, and monitor the blood pressure postoperatively, especially for the first $24 \mathrm{~h}$.

Right psoas hematoma is another complication that can often be overlooked ( 3 cases in our study). The presentation typically included dull pain on the side contralateral to the access point, most often insidious and nonspecific, with or without iliopsoas or femoral weakness. Its etiology was associated with incorrect placement of tools and contralateral annulus fibrous release damage when manipulating the discs, which always occurred on account of segmental vessel injury (34). Coronal T2-weighted imaging is used for routine preoperative evaluation to identify the aberrant vessels concerning the lumbar intervertebral space (35).

This study has a few limitations. This was a relatively small retrospective study from one clinical center. The study population was heterogeneous with different lumbar levels and pathologies. The comparative effectiveness and safety related to other interbody fusion approaches were missing and could not be determined in this study. The long-term follow-up data should be collected in the future to assess the postoperative long-term outcomes of this surgical technique.

\section{Conclusions}

OLIF is a surgical technique for mini-invasive lumbar interbody fusion and does not require neuromonitoring. This technique has a promising future. However, this approach also carries the risk of complications. By summarizing the complications encountered during treatment, we intended to make the clinicians familiar with the pros and cons. The longer follow-up radiographic and clinical data can help delineate the complication rates, the nature of cage subsidence, and the true merits of the OLIF approach.

\section{Acknowledgments}

We would like to thank Editage (www.editage.cn) for English language editing.

Funding: This study was supported by the Beijing Municipal Natural Science Foundation Project Key Project of Science and Technology Plan of Beijing Municipal Education Commission (No. KZ201910025028).

\section{Footnote}

Reporting Checklist: The authors have completed the
STROBE reporting checklist. Available at http://dx.doi. org/10.21037/atm-20-2159

Data Sharing Statement: Available at http://dx.doi. org/10.21037/atm-20-2159

Conflicts of Interest: All the authors have completed the ICMJE uniform disclosure form (available at http://dx.doi. org/10.21037/atm-20-2159). The authors have no conflicts of interest to declare.

Ethical Statement: The authors are accountable for all aspects of the work in ensuring that questions related to the accuracy or integrity of any part of the work are appropriately investigated and resolved. The study was conducted in accordance with the Declaration of Helsinki (as revised in 2013). The study was approved by the institutional research ethics committee of the Xuanwu Hospital of Capital Medical University \{No.[2018]027\}. Because of the retrospective nature of the research, the requirement for informed consent was waived.

Open Access Statement: This is an Open Access article distributed in accordance with the Creative Commons Attribution-NonCommercial-NoDerivs 4.0 International License (CC BY-NC-ND 4.0), which permits the noncommercial replication and distribution of the article with the strict proviso that no changes or edits are made and the original work is properly cited (including links to both the formal publication through the relevant DOI and the license). See: https://creativecommons.org/licenses/by-nc-nd/4.0/.

\section{References}

1. Miller C, Gulati P, Bandlish D, et al. Prepsoas oblique lateral lumbar interbody fusion in deformity surgery. Ann Transl Med 2018;6:108.

2. Phan K, Rao PJ, Scherman DB, et al. Lateral lumbar interbody fusion for sagittal balance correction and spinal deformity. J Clin Neurosci 2015;22:1714-21.

3. Rao PJ, Ghent F, Phan K, et al. Stand-alone anterior lumbar interbody fusion for treatment of degenerative spondylolisthesis. J Clin Neurosci 2015;22:1619-24.

4. Mobbs RJ, Phan K, Malham G, et al. Lumbar interbody fusion: techniques, indications and comparison of interbody fusion options including PLIF, TLIF, MI-TLIF, OLIF/ATP, LLIF and ALIF. J Spine Surg 2015;1:2-18.

5. Ozgur BM, Aryan HE, Pimenta L, et al. Extreme Lateral 
Interbody Fusion (XLIF): a novel surgical technique for anterior lumbar interbody fusion. Spine J 2006;6:435-43.

6. Davis TT, Hynes RA, Fung DA, et al. Retroperitoneal oblique corridor to the L2-S1 intervertebral discs in the lateral position: an anatomic study. J Neurosurg Spine 2014;21:785-93.

7. Hu WK, He S, Zhang S, et al. An MRI study of psoas major and abdominal large vessels with respect to the $\mathrm{X} /$ DLIF approach. Eur Spine J 2011;20:557-62.

8. Lykissas MG, Aichmair A, Hughes AP, et al. Nerve injury after lateral lumbar interbody fusion: a review of 919 treated levels with identification of risk factors. Spine J 2014;14:749-58.

9. Wang K, Zhang C, Wu H, et al. The Anatomic Characteristics of the Retroperitoneal Oblique Corridor to the L1-S1 Intervertebral Disc Spaces. Spine (Phila Pa 1976) 2019;44:E697-706.

10. Soriano-Baron H, Newcomb AGUS, Malhotra D, et al. Biomechanical Effects of an Oblique Lumbar PEEK Cage and Posterior Augmentation. World Neurosurg 2019;126:e975-81.

11. Tempel ZJ, McDowell MM, Panczykowski DM, et al. Graft subsidence as a predictor of revision surgery following stand-alone lateral lumbar interbody fusion. J Neurosurg Spine 2018;28:50-6.

12. Marchi L, Abdala N, Oliveira L, et al. Radiographic and clinical evaluation of cage subsidence after stand-alone lateral interbody fusion. J Neurosurg Spine 2013;19:110-8.

13. Silvestre C, Mac-Thiong J, Hilmi R, et al. Complications and Morbidities of Mini-open Anterior Retroperitoneal Lumbar Interbody Fusion: Oblique Lumbar Interbody Fusion in 179 Patients. Asian Spine J 2012;6:89-97.

14. Vaccaro AR, Kepler CK, Rihn JA, et al. Anatomical Relationships of the Anterior Blood Vessels to the Lower Lumbar Intervertebral Discs. J Bone Joint Surg Am 2012;94:1088-94.

15. Phan K, Mobbs RJ. Sacrum fracture following L5-S1 stand-alone interbody fusion for isthmic spondylolisthesis. J Clin Neurosci 2015;22:1837-9.

16. Kim JS, Sharma SB. How I do it? Oblique lumbar interbody fusion at L5S1 (OLIF51). Acta Neurochir 2019;161:1079-83.

17. Mehren C, Mayer HM, Zandanell C, et al. The Oblique Anterolateral Approach to the Lumbar Spine Provides Access to the Lumbar Spine with Few Early Complications. Clin Orthop Relat Res 2016;474:2020-7.

18. Benglis DM, Elhammady MS, Levi AD, et al. Minimally invasive anterolateral approaches for the treatment of back pain and adult degenerative deformity. Neurosurgery 2008;63:191-6.

19. Cummock MD, Vanni S, Levi AD, et al. An analysis of postoperative thigh symptoms after minimally invasive transpsoas lumbar interbody fusion. J Neurosurg Spine 2011;15:11-8.

20. Abel NA, Januszewski J, Vivas AC, et al. Femoral nerve and lumbar plexus injury after minimally invasive lateral retroperitoneal transpsoas approach: electrodiagnostic prognostic indicators and a roadmap to recovery. Neurosurg Rev 2018;41:457-64.

21. Aichmair A, Lykissas MG, Girardi FP, et al. An Institutional Six-year Trend Analysis of the Neurological Outcome After Lateral Lumbar Interbody Fusion. Spine (Phila Pa 1976) 2013;38:E1483-90.

22. Fujibayashi S, Kawakami N, Asazuma T, et al. Complications Associated with Lateral Interbody Fusion: Nationwide Survey of 2998 Cases During the First 2 Years of Its Use in Japan. Spine (Phila Pa 1976) 2017;42:1478-84.

23. Benglis DM, Vanni S, Levi AD. An anatomical study of the lumbosacral plexus as related to the minimally invasive transpsoas approach to the lumbar spine. J Neurosurg Spine 2009;10:139-44.

24. Uribe JS, Arredondo N, Dakwar E, et al. Defining the safe working zones using the minimally invasive lateral retroperitoneal transpsoas approach: an anatomical study. J Neurosurg Spine 2010;13:260-6.

25. Quillo-Olvera J, Lin G, Jo H, et al. Complications on minimally invasive oblique lumbar interbody fusion at L2L5 levels: a review of the literature and surgical strategies. Ann Transl Med 2018;6:101.

26. Ahmadian A, Deukmedjian AR, Abel N, et al. Analysis of lumbar plexopathies and nerve injury after lateral retroperitoneal transpsoas approach: diagnostic standardization. J Neurosurg Spine 2013;18:289-97.

27. Matsumoto T, Okuda S, Maeno T, et al. Spinopelvic sagittal imbalance as a risk factor for adjacent-segment disease after single-segment posterior lumbar interbody fusion. J Neurosurg Spine 2017;26:435-40.

28. Zeng ZY, Xu ZW, He DW, et al. Complications and Prevention Strategies of Oblique Lateral Interbody Fusion Technique. Orthop Surg 2018;10:98-106.

29. He W, He D, Sun Y, et al. Standalone oblique lateral interbody fusion vs. combined with percutaneous pedicle screw in spondylolisthesis. BMC Musculoskelet Disord 2020;21:184.

30. Pimenta L, Turner AWL, Dooley ZA, et al. Biomechanics of Lateral Interbody Spacers: Going Wider for Going 
Stiffer. ScientificWorldJournal 2012;2012:381814.

31. Kim DB, Shin MH, Kim JT. Vertebral Body Rotation in Patients with Lumbar Degenerative Scoliosis: Surgical Implication for Oblique Lumbar Interbody Fusion. World Neurosurg 2018. [Epub ahead of print]. doi: 10.1016/ j.wneu.2018.12.073.

32. Calvo-Echenique A, Cegonino J, Chueca R, et al. Standalone lumbar cage subsidence: A biomechanical sensitivity study of cage design and placement. Comput Methods Programs Biomed 2018;162:211-9.

33. Assina R, Majmundar NJ, Herschman Y, et al. First report of major vascular injury due to lateral transpsoas approach

Cite this article as: Cheng $\mathrm{C}$, Wang $\mathrm{K}$, Zhang $\mathrm{C}$, Wu H, Jian F. Clinical results and complications associated with oblique lumbar interbody fusion technique. Ann Transl Med 2021;9(1):16. doi: 10.21037/atm-20-2159 leading to fatality. J Neurosurg Spine 2014;21:794-8.

34. Beckman JM, Vincent B, Park MS, et al. Contralateral psoas hematoma after minimally invasive, lateral retroperitoneal transpsoas lumbar interbody fusion: a multicenter review of 3950 lumbar levels. J Neurosurg Spine 2017;26:50-4.

35. Orita S, Inage K, Sainoh T, et al. Lower Lumbar Segmental Arteries Can Intersect Over the Intervertebral Disc in the Oblique Lateral Interbody Fusion Approach with a Risk for Arterial Injury. Spine (Phila Pa 1976) 2017;42:135-42. 